\title{
Wie man einen Kuss verwahrt: Die Porträtminiatur als intimes Andenken
} Hanneke Grootenboer

Um 1800 wurde, wahrscheinlich in St. Petersburg, eine Serie von drei sehr ungewöhnlichen Bildern gemalt, die sich noch heute in der Sammlung der Eremitage befindet (Abb. 1-3). ${ }^{1}$ In ähnlichen Kompositionen zeigen diese Miniaturen isolierte Partien eines menschlichen Körpers: ein einzelnes Auge, einen Mund sowie zwei Hände mit einem Schriftstück, jeweils von blaugrauen Wolken umgeben. Die mit relativ preiswerten bronzenen Rahmen versehenen kleinen Bildanhänger sind nicht signiert, und es ist praktisch unmöglich, in diesen Darstellungen eine porträtierte Person zu identifizieren.

In seiner frühen, 1824 erschienenen Biografie des Miniaturmalers Gerhard von Kügelgen (1772-1820) beschreibt Friedrich Hasse die zu Beginn des 19. Jahrhunderts in St. Petersburg verbreitete Mode, an der Brust ein Medaillon zu tragen, das ein Augenporträt mit einer Haarlocke enthielt. ${ }^{2}$ Auch wenn die hier gezeigten drei Bildanhänger kein Haar einschlossen, waren sie wahrscheinlich Teil dieser Mode. Von Kügelgen, ein sehr erfolgreicher Künstler am königlichen Hof, schuf nachweisbar mindestens zwei Augenminiaturen, darunter ein Augenporträt von Fürst Adam Jerzy Czartoryski, das auch von Hasse erwähnt wird, ${ }^{3}$ sowie das bekanntere Selbstporträt, das sich auf der Rückseite des Porträts von Wilhelm Johann Zoege von Manteuffel befindet (Abb. 4). Man kann durchaus annehmen, dass er auch Miniaturen in der Art der St. Petersburger Bildserie produzierte. Die drei Darstellungen sind offensichtlich als Ensemble konzipiert, und es liegt nahe, dass das Auge, die Lippen und die Hände zum selben Modell gehörten. Es ist unklar, ob es sich hierbei um ein Mitglied der wohlhabenden Familie Jussupow handelt, in deren Sammlung sich die Objekte befanden, bevor sie in die Eremitage gelangten; die Miniaturen könnten auch

\footnotetext{
As there are some (translated) parts of this article which have been published in English in your article on "Eye, Mouth and Hand Miniatures" in the book „La miniature en Europe“, it would possibly be an option to mention this in a note at the beginning of the footnotes? Could you contact the editors (Éditions du CEREMIF, Paris) with regard to the copyright and inform us on the conditions? Some shorter passages in this article are based on your book „Treasuring the Gaze“, maybe you could also check and decide on this case?
cherter passages in this artic

${ }^{1}$ Vgl. zu der folgenden Bildanalyse auch Hanneke Grootenboer, Intimate Portraits: Eye, Mouth and Hand Miniatures, in: Nathalie Lemoine-Bouchard (Hg.), La miniature en Europe. Des portraits de propagande aux œuvres éléphantesques, Paris 2013, S. 104-108, hier 104.

2 ,Es war damals Sitte in Petersburg, ein gemaltes Auge, mit einer Locke von dem Haare der Person eingefaßt, unter Glas auf der Brust zu tragen.“ Friedrich Christian August Hasse, Das Leben Gerhards von Kügelgen, Leipzig 1824, S. 77, Anm.

${ }^{3}$ Ebd., S. 77.
}

Commented [MV1]: I added this part with Wilhelm Johann Zoege von Manteuffel: is this ok? (You wrote: "and the better-known self-portrait we see here") 
ein Geschenk an die Familie gewesen sein. In jedem Fall aber muss es sich bei dem Ensemble um eine äußerst private Gabe an eine bestimmte Person gehandelt haben, die eine sehr spezifische Botschaft trug. Es stellt sich die Frage, welche Bedeutung diese Objekte, diese Launen der Miniaturmalerei hatten. Sind sie Porträtminiaturen grundsätzlich ähnlich oder tatsächlich etwas ganz anderes? Zumindest können sie, auch wenn es sich hier um die Porträts eines Auges und eines Mundes handelt (bezüglich der Hände bin ich mir hier weniger sicher), nicht als Bildnisse im konventionellen Sinne bezeichnet werden. ${ }^{4}$

Betrachten wir die Objekte zunächst einzeln etwas näher. Augenminiaturen sind, wie wir wissen, ungewöhnlich, jedoch nicht unüblich - so sind etwa 200 Beispiele aus dem späten 18. und frühen 19. Jahrhundert in öffentlichen und privaten Sammlungen erhalten (Abb. 5). Zunächst kamen sie bei der englischen Aristokratie in Mode, nachdem der Prince of Wales bei einem Opernbesuch ein Medaillon mit einem gemalten Auge seiner inoffiziellen Gattin Maria Fitzherbert an einem Armband getragen hatte. ${ }^{5}$ Ende der 1780er-Jahre waren sie in London bereits recht verbreitet, und von dort ausgehend entwickelte sich dieser Trend im ersten Jahrzehnt des 19. Jahrhunderts auch in den aristokratischen Kreisen Kontinentaleuropas. ${ }^{6}$ So ist etwa überliefert, dass Marie-Louise von Österreich, die zweite Ehefrau Napoleons I., eine beträchtliche Sammlung besaß; ${ }^{7}$ Emma Hamilton und Horatio Nelson tauschten Augenporträts aus, und auch einige Augen Byrons zirkulierten in den 1810er-Jahren. ${ }^{8}$ Galten Augenporträts zunächst als romantisches Liebespfand - als Bild eines Blickes einer geliebten Person, der stetig auf dem Betrachter ruhte - , so dienten sie später auch als allgemeinere, jedoch stets intime Gabe. Ganze Familien ließen offenbar ihre Augen malen: Dies wird zum Beispiel an einem 1802 als Geburtstagsgeschenk für König Friedrich Wilhelm III. geschaffenen ,Augen-Gruppenporträt‘ ersichtlich, das

${ }^{4}$ Vgl. Grootenboer 2013 (wie Anm. 1), S. 104.

${ }^{5}$ Richard Cosway, Augenporträt von Mrs. Fitzherbert, ca. 1786, Aquarell auf Elfenbein,

Privatsammlung. Vgl. dazu auch Hanneke Grootenboer, Treasuring the Gaze: Eye Miniature Portraits and the Intimacy of Vision, in: The Art Bulletin 88 (2006), Nr. 3, S. 496-507, hier 496-498; Hanneke Grootenboer, Treasuring the Gaze: Intimate Vision in Late Eighteenth-Century Eye Miniatures, Chicago/London 2012, S. 45-47.

${ }^{6}$ Vgl. Grootenboer 2013 (wie Anm. 1), S. 104; Grootenboer 2006 (wie Anm. 5), S. 496; Grootenboer 2012 (wie Anm. 5), S. 48-62. Vgl. auch Astrit Schmidt-Burkhardt, Sehende Bilder: Die Geschichte des Augenmotivs seit dem 19. Jahrhundert, Berlin 1992, S. 19-21.

${ }^{7}$ Vgl. Grootenboer 2012 (wie Anm. 5), S. 61; Hubert Glaser (Hg.), Krone und Verfassung. König Max I. Joseph und der neue Staat (Ausst.-Kat. München, Staatliches Museum für Völkerkunde), München 1980, S. 613.

${ }^{8}$ Vgl. Grootenboer 2012 (wie Anm. 5), S. 72; 164-168; Grootenboer 2006 (wie Anm. 5), S. 501.

Commented [MV2]: Would this be ok?

(You wrote "an image of a loved one's gaze, continuingly resting upon you as if it were a kind of camera".) I would suggest to delete the comparison to the camera in order to further underline the perspective of the $18^{\text {th }}$-century viewer? 
die Augen seiner Ehefrau Königin Louise von Preußen und von vier ihrer Kinder zeigt (Abb. 6). ${ }^{9}$

Vielleicht resultierte der größte Reiz der Augenporträts aus ihrer Anonymität: Dass die Identität des Dargestellten nur dem Besitzer des Augenporträts bekannt war, niemals aber der Außenwelt, verstärkte die Einzigartigkeit dieses Geschenks. Der oder die Liebende konnte sich am Bild eines Blickes erfreuen, dessen Ursprung nur er oder sie kannte. Manche Augen wurden auch zum Trauerschmuck, indem Kristalle oder Diamanten als symbolische Darstellung von Tränen auf dem elfenbeinernen Träger angebracht wurden (Abb. 7). Vor dem Hintergrund der Kultivierung des Weinens im Zeitalter der Empfindsamkeit, als Tränen als gegenseitiger Gabentausch betrachtet wurden, scheint das hier gezeigte gemalte Auge diamantene Tränen über seinen eigenen Tod zu weinen, während es zugleich eine unmittelbare Reaktion seines Betrachters in Form tatsächlicher Tränen hervorrufen soll. ${ }^{10}$

Im Unterschied zu den einst sehr modischen Augenminiaturen sind Mundminiaturen extrem selten. Wir wissen mit Sicherheit, dass der bekannte Miniaturenmaler Richard Cosway einmal auch die Lippen des Prince of Wales malte, als er verschiedene Augenporträts von ihm und Maria Fitzherbert schuf. ${ }^{11}$ Das St. Petersburger Medaillon gehört damit zu den wenigen erhaltenen Beispielen von Mundminiaturen, und man kann sich fragen, um was für eine Darstellung es sich hier eigentlich handelt. Ist es lediglich ein Porträt, oder trägt das Bild noch eine andere Bedeutung? Johann Caspar Lavater schrieb in seinen Physiognomischen Fragmenten, „[a]lles liegt in dem menschlichen Munde, was im menschlichen Geiste liegt“, und der Mund scheint ihm so noch charaktervoller als das Auge. ${ }^{12}$ Während das Auge Bilder unserer verborgenen Gemütserregungen male und als solches mehr zur Seele gehöre, betont

\footnotetext{
${ }^{9}$ Vgl. dazu Grootenboer 2012 (wie Anm. 5), S. 53-54.

${ }^{10}$ Grootenboer 2013 (wie Anm. 1), S. 104. Vgl. auch ausführlicher Grootenboer 2012 (wie Anm. 5), S. 89-111.

${ }^{11}$ Diese Objekte wurden in einer Liste mit ausstehenden Forderungen erwähnt. Zum Inventar, in dem sich diese Liste befindet, vgl. Stephen Lloyd, The Cosway Inventory of 1820: Listing Unpaid Commissions and the Content of 200 Stratford Place, Oxford Street, London, in: The Volume of the Walpole Society 66 (2004), S. 163-218, hier 171; 197.

12 Johann Caspar Lavater, Physiognomische Fragmente, zur Beförderung der Menschenkenntnis und Menschenliebe, Dritter Versuch, Leipzig/Winterthur 1777, S. 121.
}

Commented [MV3]: You wrote "and it is fair to say that you are looking at one of the very few surviving examples." You also wrote in the sentence after next, "What does this mouth actually represent?" I guess, in both sentences you mean the Petersburg mouth miniature? (In your original power point presentation of the talk, you showed the Petersburg mouth portrait at this point, after the eye portrait with tears). I would therefore suggest, in order to keep the flow of the text, to put these parts together (see the third sentence of this passage, marked in yellow). In this way, you first have the general statement about mouth portraits, then the example of Cosway and then again the specific case of Petersburg.

Commented [MV4]: I have put "as the items were mentioned in a list of outstanding debts" in the footnote, see below.

Commented [MV5]: You wrote: "or is there a deeper meaning behind this depiction?". I would suggest to write "oder trägt das Bild noch eine andere (= another) Bedeutung?". In this way, it is more open than "deeper", thereby not excluding that also a portrait could have a deeper meaning. 
Lavater im Hinblick auf den Mund: „Wie die Lippen, so der Charakter.“13 Könnte das St. Petersburger Bild also letztlich als ein Bild des Charakters einer bestimmten Person betrachtet werden? Tatsächlich mag die Mode der Augen- oder Mundporträts zum Teil von Lavaters Theorie geprägt gewesen sein, dass das Wesen eines Menschen in den einzelnen Teilen des Gesichts gelesen werden könne. So könnten wir uns etwa fragen, was für geheime Gemütsbewegungen oder Charakterzüge wir in einem sehr ausgefallenen Gruppenporträt im Innern einer Schnupftabakdose entdecken können, das verschiedene Lippen- und Augenpaare vereint (Abb. 8). Es ist schwer, sich dem Eindruck zu entziehen, dass diese Augen und Lippen eine Sammlung verbotener Blicke und im Verborgenen zugeworfener Küsse sein könnten, die das Behältnis zu einem köstlichen geheimen Souvenir eines Libertins machten. Mit Blick auf diese ungewöhnliche Schnupftabakdose stellt sich bei dem Mund der St. Petersburger Miniatur die Frage, ob er tatsächlich die Gesichtspartie einer bestimmten Person abbildet oder ihren Charakter bezeichnet - oder ob er nicht vielmehr das Porträt eines Kusses ist, der ebenso sehr einen künftigen Kuss wie auch einen bereits geschehenen Kuss assoziiert? ${ }^{14}$

Die etwas größere dritte Miniatur des St. Petersburger Ensembles schließlich zeigt zwei Hände und ein Schriftstück mit den Worten „La vraie Amitié est le don des Dieux“ („Wahre Freundschaft ist ein Geschenk der Götter“) und ist meines Wissens einzigartig in der Geschichte der Miniaturmalerei. Wissentlich oder unwissentlich wird hier das Handmotiv einer viel älteren Miniaturtradition aufgegriffen, wie wir es in Nicholas Hilliards Porträt eines unbekannten Mannes von 1588 sehen können (Abb. 9). ${ }^{15}$ Auf diese Weise bietet das dritte St. Petersburger Bild meines Erachtens einen Schlüssel zur Bedeutung und Funktion der anderen beiden Bilder und fasst zudem das grundsätzliche Wesen der Anredestruktur von Porträtminiaturen zusammen. Hände greifen und halten, sie ergreifen und halten fest, sie geben und nehmen. Die Miniatur scheint gleichsam zum Ausdruck bringen zu wollen, dass sie

\footnotetext{
13 Johann Caspar Lavater, Physiognomische Fragmente, zur Beförderung der Menschenkenntnis und Menschenliebe, Vierter Versuch, Leipzig/Winterthur 1778, 260. Siehe hier und im Folgenden auch Grootenboer 2013 (wie Anm. 1), S. 106.

${ }^{14}$ Neben Porträtminiaturen von Augen und Mündern sollen auch Brüste ,im Kleinen` verewigt worden sein. So wurden Brustminiaturen manchmal an der Deckelinnenseite von Schnupftabakdosen angebracht, um eine sehr persönliche Gabe daraus zu machen. Derartige außerordentlich private Bilde wurden entsprechend geschützt, und es existieren nur wenige bekannte Objekte. Could you add a bibliographical reference?

${ }^{15}$ Vgl. hier und im Folgenden ebenfalls Grootenboer 2013 (wie Anm. 1), S. 106.
}

Commented [MV6]: You wrote "in a snuffbox" - just to check: is the picture inside or is it on the outside of the box?

Commented [MV7]: See also illustration check list (powerpoint file): Could you provide us with the complete caption of this picture

(artist, title, date, technique, dimensions, location)?

Commented [MV8]: I have three questions:

1) You mentioned breast portraits at this point ("In addition to portrait miniatures of eyes and mouths, breasts, too, are said to have been eternalized "in the little," sometimes set in the inside lids of snuff boxes to create a highly personal gift. Such extremely private images have been duly protected, such that I am not even able to show you one today"). For the written paper, in contrast to the talk, I would suggest (in order to keep the focus) to put this remark in the footnote as an addition; I would also suggest to delete "I am not even able to show you one today".

2) Could you add a bibliographical reference to breast portraits in the footnote?

3) Is Goodridge's painting, which you discuss later, part of this category you mention here? Or is this a different case? Would it make sense to make a reference to your later passage? 
sich mit der Freundschaftserklärung auf dem Schriftstück selbst in die Hände des Empfängers legt und verlangt, festgehalten, sicher verwahrt, ja als Schatz gehütet zu werden. Die dunklere, breitere und maskuliner erscheinende linke Hand, die gerade die Botschaft geschrieben hat, ist offenkundig die Hand des Schenkenden, während die elegante, längliche und feminin erscheinende Hand das Papier entgegennimmt. Das Schriftstück geht somit in andere Hände über, gleich einer Miniatur, die verschenkt wird.

Ich möchte diese drei außergewöhnlichen Miniaturen als Ausgangspunkt für einige Reflexionen über das Wesen der Porträtminiatur nehmen. Gerade die Tatsache, dass Augen- und Mundporträts in den Randbereichen der Kunstgeschichte überlebt haben, als Sammlerobjekte und in den unteren Schubfächern der Museumsarchive und (daher) in kunsthistorischen und bildwissenschaftlichen Studien wenig theoretisiert worden sind, kann sich für uns als Vorteil erweisen. Als vergessene Artefakte konfrontieren sie uns als Kunsthistoriker mit einem ungewohnten Umgang mit Miniaturen. So könnten die winzigen Augen- und Mundporträts, die plötzlich in Mode kamen und wieder in Vergessenheit gerieten, auf einen Verdichtungsmoment in der Entwicklung der Geschichte der Kunst verweisen, der aus unserem Blickfeld verschwunden ist. Diesen Moment können wir rekonstruieren, wenn wir Kommunikationsweisen und Ausdrucksstrategien offenlegen, die bisher unsichtbar geblieben sind. Wie wir sehen werden, fasst die St. Petersburger Miniatur mit dem Schriftstück das Wesen der Anredestruktur zusammen, das die Porträtminiatur im Allgemeinen wie auch die Mund- und Augenminiaturen gemeinsam haben. ${ }^{16}$ Ausgehend von diesem spezifischen Objekt möchte ich versuchen, den Knoten in der Geschichte der Miniaturmalerei zu entwirren.

In diesem Beitrag betrachte ich die Porträtminiatur mehr als evokatives Objekt denn als bloßes Bildnis. Tief verwurzelt in komplexen Ökonomien des Tausches - des Übergehens in andere Hände - ist sie immer eine Botschaft wie auch eine Antwort, ein Geschenk wie auch eine Einladung; ganz ähnlich wie das Schreiben eines Briefes beantwortet sie eine bestimmte Handlung und erwartet zugleich eine weitere. Es ist eine Kultur geheimer Botschaften, gestohlener Blicke und versprochener Küsse, in

\footnotetext{
${ }^{16}$ Ebd.
}

Commented [MV9]: You wrote "these peculiar minipaintings of eyes and lips" - I replaced it with "sie" (they) because we have the "winzigen Augen- und Mundporträts" (tiny portraits of eyes and mouths) in the next sentence "dense moment"? Is it a moment of density or a blind spot? 
der die Augen- und Mundporträts zu verorten sind. Diese unterscheiden sich, so möchte ich behaupten, von Gesichtsdarstellungen, wenngleich sie in der Kunst der Miniaturmalerei verwurzelt sind: Die isolierten Körperpartien sind keine Synekdochen, sie stehen nicht für das Gesicht als Ganzes, sondern sie sind vielmehr ein vergegenständlichtes Gefühl: ein Kuss oder Blick, der greifbar gemacht worden ist. ${ }^{17}$

Um diese Art des Austausches wirklich zu verstehen, betrachten wir zunächst Pompeo Batonis bekanntes Doppelporträt von Sir Sampson Gideon und einem unbekannten Freund von 1767, das ein musterhaftes Beispiel für die Anredestruktur einer Miniatur bietet (Abb. 10). ${ }^{18}$ Gideon sitzt an einem Tisch, auf dem eine MinervaBüste zu sehen ist, und hält seinem Freund ein Medaillon zur Ansicht entgegen, das ein Miniaturporträt und eine Haarlocke enthält. Dabei dürfte es sich um ein Bildnis der späteren Gattin Gideons, Maria Eardley Wilmot, handeln, das Gideon als Bestätigung der gemeinsamen Verlobung empfangen hat. ${ }^{19}$ Sein Freund blickt das Bildnis intensiv an und erscheint gedankenversunken. Vergeblich versucht der Hund, seine Aufmerksamkeit zu erregen. Das Medaillon befindet sich genau in der Mitte der Bildkomposition und zugleich auch im Zentrum der Bilderzählung: der Transaktion zwischen den beiden Protagonisten.

Susan Stewart hat bekanntermaßen argumentiert, dass die Miniatur am Beginn der privaten, individuellen Geschichte stehe. ${ }^{20}$ Indem Batonis Gemälde darstellt, wie Gideon das Medaillon öffnet und das darin verborgene Porträt seinem Freund zeigt, macht es einem intendierten Publikum den privaten Blick, die private Geschichte des Geschenks öffentlich. Das Gemälde erzeugt den Eindruck der Anrede, vermittelt durch narrative Spannung: zwischen dem Bild und Gideon, zwischen Gideon und seinem Freund wie auch zwischen dem Freund und dem Hund. Die ernsten Gesichter

\footnotetext{
${ }^{17}$ Ebd.

${ }^{18}$ Für eine ausführlichere Diskussion dieses Gemäldes vgl. Angus Trumble, A Roman Holiday Pompeo Batoni and Sir Sampson Gideon, in: Art and Australia 36 (1998), Nr. 1, S. 84-87; vgl. auch Edgar Peters Bowron und Peter Björn Kerber (Hg.), Pompeo Batoni: Prince of Painters in EighteenthCentury Rome (Ausst.-Kat. Houston, Museum of Fine Arts, London, The National Gallery), New Haven/London 2007, S. 69. Siehe zu dem Porträt ebenfalls Grootenboer 2012 (wie Anm. 5), S. 25-26 und Marcia Pointon, “Surrounded with Brilliants": Miniature Portraits in Eighteenth-Century England, in: The Art Bulletin 83 (2001), Nr. 1, S. 48-71, hier 65-66.

${ }^{19}$ Vgl. Bowron und Kerber 2007 (wie Anm. 18), S. 69.

${ }^{20}$ Susan Stewart, On Longing: Narratives of the Miniature, the Gigantic, the Souvenir, the Collection, Durham/London 1993, S. 71.
} 
der beiden Dargestellten, Gideons besorgter Blick, den er dem Freund zuwirft, und der abgewandte Blick der Minerva-Skulptur suggerieren eine erzählerische

Auflösung. Der Schlüssel zur zentralen Position der Miniatur in dieser gemalten Erzählung und zum besonderen Kontrast zwischen ihrer sehr privaten Natur und ihrer allgemeinen Offenlegung liegt, wie ich denke, in der Beziehung des Porträts zu dem Brief, den Gideon in der linken Hand hält. Der Brief ist vom Medaillon ebenso weit entfernt wie der Kopf des Freundes, sodass das Medaillon von beidem sozusagen eingerahmt wird. Während wir die Porträtminiatur hier buchstäblich in andere Hände übergehen sehen, ist es zugleich bemerkenswert, dass das Medaillon Gideon von seiner Verlobten nicht persönlich übergeben worden ist, sondern in einem Brief an ihn gesandt wurde. Trotz der Bedeutung, die das Medaillon für das Paar besitzt, geht es in dem Gemälde weniger um den Austausch der Miniatur zwischen den Liebenden als um die Sichtbarkeit der Miniatur für die erwarteten Betrachter des Bildes. Die Miniatur dient hier als bedeutsamer Zusatz zum Brief, dem sie beigefügt ist. Wir könnten sogar sagen, dass die Miniatur, eben weil sie Gideon nicht persönlich überreicht wurde, sich eher an ihn wendet, als dass sie ihm geschenkt wird. ${ }^{21}$

Die Praxis des Betrachtens aus nächster Nähe in relativer Abgeschiedenheit, die die Miniatur verlangt, steht in scharfem Kontrast zu der exzessiven Sichtbarkeit, die Batoni dem Medaillon verleiht, indem er es zum Fokus seiner großen Komposition macht. Aber wer, fragt man sich, interessiert sich dafür, die Übergabe des Medaillons der zukünftigen Braut in so monumentaler Größe zu sehen, abgesehen von den beiden Protagonisten dieser Gabengeschichte? Batoni legt derartiges Gewicht auf die Überreichung des Medaillons, und doch bleibt unklar, für wen sie eigentlich in Szene gesetzt wird.

Wie Marcia Pointon bemerkt, legt die Analogie zwischen der Tauschökonomie der Miniaturporträts und der Intimität des Briefeschreibens einen Gefühlsdiskurs offen, in dem die Porträtminiatur nicht einfach das Geschenk einer geliebten Person ist, sondern Teil einer komplexen Konstellation imaginärer Gesten und Erwiderungen. ${ }^{22}$ Wie ein Brief nahezu immer in Erwartung einer Antwort versandt wird, setzt auch die Anredestruktur der Miniatur eine Antwort, eine Erwiderung voraus. In Batonis

${ }^{21}$ Vgl. auch Grootenboer 2012 (wie Anm. 5), S. 26.

${ }^{22}$ Pointon 2001 (wie Anm. 18), S. 63-67.
Commented [MV11]: You wrote: "The letter is at equal length from the locket and the companion's head, framing it as it were." I guess you mean that the locket is framed by the head and the letter? 
Gemälde sehen wir, dass die Miniatur als Bestätigung der Verlobung tatsächlich eine Art Erwiderung ist. Aber ihre postalische Ankunft erwartet ihrerseits eine Antwort.

Genau genommen wird diese ganze Transaktion von Botschaften, die in andere Hände übergehen, in der St. Petersburger Miniatur der beiden Hände mit dem Schriftstück zusammengefasst. Wenn wir für einen Augenblick annehmen, dass Porträtminiaturen tatsächlich, ganz ähnlich wie schriftliche Korrespondenz, Teil einer solchen Tauschökonomie der Gefühle waren, stellt sich die Frage, welche Botschaft das Ensemble der drei Miniaturen vermittelte. Bilder wie Francis Wheatleys erotisch gefärbte Komposition, die die intime Beziehung einer jungen Frau zu einer Porträtminiatur thematisiert, suggerieren, dass diese Porträtobjekte nicht allein Gebrauchsgegenstand waren oder der Ergötzung dienten, sondern dass sie vielmehr Teil einer fortwährenden ,Konversation“ oder ,Korrespondenz‘ waren (Abb. 11). Eine Geliebte Lord Byrons, Lady Frances Webster, schrieb 1813 in einem Brief an den Dichter, dass sie sich so an die Gegenwart seines Bildes gewöhnt habe, dass es eine Art Freund und gar ein Gesprächspartner für sie geworden sei. Das Bild „[...] seems to speak to me-it speaks comfort to my wounded mind [...] I cannot put it out of my sight [...].“23 In einem weiteren Brief desselben Jahres hebt sie hervor: „Your picture dearest Byron is my constant Companion-I gaze at it — till every feature seems to speak. “24

Im Gegensatz zu Porträtminiaturen, die als Bildnisse bewundert, gehalten, an die Brust gedrückt und geküsst werden können, scheinen Augen- oder Mundporträts nicht zu Zärtlichkeiten einzuladen. Und dennoch scheinen sie etwas mit dem oder der Betrachtenden zu ,machen‘, wenn sie seinen oder ihren Blick erwidern. Wenn wir auf eine um 1800 entstandene Augenminiatur blicken, müssen wir zugeben, dass es dort kaum etwas zu sehen gibt (Abb. 12). Es passiert sehr wenig im Hinblick auf das Augenporträt als Bild, und dennoch wird viel gesehen - aber eher auf der Seite des kleinen Bildes als auf der Seite des Betrachters. Das Augenporträt ist nicht bloß ein Sehobjekt, eine Darstellung, die betrachtet werden kann, sondern es scheint selbst

\footnotetext{
${ }^{23}$ Brief von Lady Frances Webster an Lord Byron, 25. November 1813, zit. in: Annette Peach, Portraits of Byron, in: The Volume of the Walpole Society 62 (2000), S. 48.

${ }^{24}$ Brief von Lady Frances Webster an Lord Byron, 28. Dezember 1813, zit. in: Peach 2000 (wie Anm. 23), S. 48.
} 
eine Art Sicht zu besitzen. Es stellt sich die Frage, wer genau hier schaut. Sind wir es, die die kleinen Bilder anblicken, oder sind diese gespenstischen Augen nur dazu da, uns anzusehen? Ist das Einzige, was tatsächlich in diesen winzigen Bildern geschieht, die Erwiderung unseres Blickes? Wie beim Briefeschreiben gibt es hier ein deutliches Moment der Symmetrie, indem das Augenporträt - immer - einen flüchtigen Blick beantwortet und dabei stets das zurückgibt, was der Betrachter ihm schenkt, nämlich den Blick. Sollten wir die Augenminiatur vielleicht nicht als Bildnis im traditionellen Sinn verstehen? Ist sie nicht vielmehr eine Liebesgeste, eine fortwährende Antwort auf eine vorangegangene Frage und eine Bitte um Erwiderung, eine Art von Zwinkern oder visuellem Kuss, der uns in dem Moment zugeworfen wird, wenn wir den Blick darauf richten? ${ }^{25}$

Um 1800 halten viele Porträtierte in Porträtminiaturen ganz bewusst Briefe in ihren Händen, genauso wie in Darstellungen früherer Jahrzehnte Porträtierte eine Porträtminiatur festhielten. Wenn wir Miniaturen mit Briefen näher betrachten, erhalten wir eine tiefere Einsicht in die Art dieser Korrespondenzen. Als eines von vielen Beispielen zeigt ein Bild des französischen Künstlers Jean Baptiste Soyer eine unschuldig lächelnde junge Frau vor dem stilisierten Hintergrund einer romantischen Landschaft (Abb. 13). ${ }^{26}$ Ihr linker Arm stützt sich auf einen Felsen, ihre linke Hand, an deren Ringfinger ein goldenes Band zu sehen ist, ist ausdrucksvoll in der Mitte des Bildes positioniert. Mit der rechten Hand umklammert sie einen Brief, den sie soeben erhalten zu haben scheint - das rote Siegelwachs ist noch auf dem Papier zu sehen. Ihre offenen Augen und ihr fröhliches Lächeln scheinen uns nach langer Abwesenheit zu begrüßen, und wir Betrachtenden mögen uns an dieser Begrüßung erfreuen, die unseren Blick empfängt - bis wir langsam gewahr werden, dass der weiche Blick und das entzückende Lächeln sich gar nicht an uns richten, sondern an die Person, deren Brief sie gerade gelesen hat. Völlig darauf eingestellt, gesehen zu werden, in einer Art, die ebenso narzisstisch ist wie gefällig, strecken sich die Augen und sogar der ganze Körper der jungen Frau aus, um den imaginären Blick der geliebten Person zu erwidern, an die das Bild gerichtet ist. Die Botschaft in diesem Bild wird durch ihre Körpersprache geschrieben. Ihr Lächeln und ihr intensiver Blick verraten Begehren

\footnotetext{
${ }^{25}$ Grootenboer 2013 (wie Anm. 1), S. 107.

${ }^{26}$ Vgl. zu Soyers Miniatur im Folgenden auch meine Bildanalyse in Grootenboer 2012 (wie Anm. 5), S. 26-28.
} 
oder sogar ein Ausgeliefertsein im Hinblick auf eine Antwort auf diesen Brief. Das Bild ist somit ihre Antwort. Und zugleich bleibt dieser Brief unlesbar für jeden mit Ausnahme des intendierten Bildbetrachters. Ganz gleich, wie sehr unser voyeuristischer Blick sich in dieses kleine Bild versenkt, die Freigiebigkeit dieser Frau besteht in einem Geschenk, das genau genommen nicht jedem gemacht werden kann. Was sie mit diesem Bild zu geben bereit ist, ist eine Art Kontakt oder Berührung über die Entfernung hinweg. Die Porträtminiatur ist nicht nur das kostbare Geschenk eines schönen Bildnisses, das bewundert werden soll, sondern ein Träger, der einen besonderen Raum erzeugt, eine intime Umgebung, in der die Dargestellte und die von ihr angesprochene Person auf gewisse Weise miteinander allein sind. ${ }^{27}$

Soyers Miniatur und andere dieser Art sind augenfällige Beispiele, in denen Briefe und Bilder im Rahmen einer Korrespondenz austauschbar werden. Der durch die exklusive Anredestruktur entworfene intime Rahmen, durch den das Porträt als Botschaft einen privaten Betrachter voraussetzt, wird noch evidenter in einem deutschen, von einem unbekannten Künstler geschaffenen Miniaturporträt der Gräfin Edling von etwa 1785 (Abb. 14). Gräfin Edling entschied sich, die Risiken einer deutungsoffenen visuellen Kommunikation zu vermeiden. Um ihre Botschaft ohne jegliche Zweideutigkeit zu vermitteln, hält die Dargestellte ein Schriftstück in den Händen, auf dem eine handgeschriebene Botschaft an den intendierten Empfänger des Geschenks zu lesen ist: „Lebe lang und leb gesund wünscht mein Herz und spricht mein Mund.“ Trotz ihrer Bemühung, sich unmissverständlich auszudrücken, findet sich die Gräfin mit dem Paradox konfrontiert. Sie möchte über den Text einen Wunsch einfügen, der nicht im Sichtbarkeitsbereich der Miniatur zum Ausdruck gebracht oder ausgesprochen werden kann. Jedoch steht dieser Text, der artikulieren soll, was sie sagen möchte, im Widerspruch zu ihrem Bildnis, denn ihr fest geschlossener Mund spricht eben gerade nicht. Es scheint, als ob die hier versuchte Vereinigung von Porträt und Brief den Wunsch der Gräfin zeigt, den Gegensatz von Wort und Bild zu überwinden. ${ }^{28}$ Diesen Gegensatz legt Chevalier Danceny der Marquise de Merteuil in Pierre Choderlos De Laclos’ Briefroman Les Liaisons dangereuses treffend offen: „Mais une lettre est le portrait de l’âme. Elle n’a pas,

\footnotetext{
${ }^{27}$ Ebd., S. 30.

${ }^{28}$ Vgl. zu dieser Bildanalyse auch Grootenboer 2012 (wie Anm. 5), S. 30-31.
}

Commented [MV13]: Is this ok? I checked this picture at the website of the Tansey Collection and it says here that her name is Edling, not Erdin, see: http://tansey$\underline{\text { miniatures.com/sammlung-2/\#/11016 }}$ 
comme une froide image, cette stagnance si éloignée de l’amour [...].“29 Gräfin Edling wie auch Danceny waren wahrscheinlich von einer berühmten Passage aus dem bekannten mittelalterlichen Briefwechsel zwischen Héloïse und Abélard inspiriert, in dem Héloïse an ihren Geliebten schreibt: „Si les portraits de nos amis absens ravivent leur souvenir et allègent le regret de leur absence par une vaine et trompeuse consolation, combien sont plus précieuses les lettres qui nous apportent de véritables empreintes de l’ami absent!“30

Porträtminiaturen mit Briefen sind, wie ich vorschlagen möchte, selbstreflexive Versuche, die Porträts von Gesicht und Herz zu vereinen, um ein vollständigeres Selbst-,,Bild“ zu erzeugen, das in Erwiderung ebenso wie in Erwartung einer Liebesbezeugung geschenkt werden kann. Wenn die Briefe in diesen Porträtminiaturen inspirieren und „sprechen“ sollen, können wir uns fragen, welche Art der intimen Kommunikation eine Frau in einer von François Dumont um 1800 gemalten Porträtminiatur führt (Abb. 15): Die junge Frau stützt ihren Kopf in einer nachdenklichen Geste auf ihre Hand und sieht gerade von einem Brief auf, der neben ihr auf dem Tisch liegt, um dem Empfänger des Bildes direkt in die Augen zu blicken. Mit einer Freimütigkeit, die an die Frau in Soyers Bild erinnert, macht ihr Körper eine Aussage, ihre Offenheit gegenüber dem Adressaten ist jedoch deutlich stärker sexuell geprägt. Die Nähe zwischen ihrer linken Brust und dem Brief deutet eine Angebotsgeste an; die Bänder ihres Kragens lösen sich bereits und entblößen einen Teil ihrer Brust. Es scheint in der Tat so, als sei ihre Antwort die Einladung zu einer intimen Begegnung, das Versprechen, ebendiese Brust zu sehen, vielleicht zu berühren. ${ }^{31}$ Es existieren einige Miniaturen dieser Art, die, so könnte man resümieren, weniger einen voyeuristischen Blick auf die Brust einer jungen Frau bieten als vielmehr das Versprechen einer Berührung über die Entfernung hinweg. ${ }^{32}$

\footnotetext{
${ }^{29}$ Pierre Choderlos de Laclos, Les Liaisons Dangereuses, hg. von René Pomeau, Paris 1981 (Erstausgabe Paris 1782), S. 338.

${ }^{30}$ Héloïse et Abélard, Lettres. Traduction nouvelle par Le Bibliophile Jacob, précédée d'un travail historique et littéraire par M. Villenave. Paris 1880 (Erstausgabe Paris 1840), S. 125.

${ }^{31}$ Vgl. auch Grootenboer 2013 (wie Anm. 1), S. 107.

${ }^{32}$ Vgl. zum Beispiel die noch explizitere Miniatur von Philip Jean von ca. 1785 und die Miniatur von Joseph Marie Bouton (ca. 1800). Could you provide us with more information on these two pictures (title, location)?
}

Commented [MV14]: See also illustration check list (powerpoint file): Could you provide us with the complete caption of the picture?

Commented [MV15]: You wrote: “And I give you two, more explicit, examples, by Philip Jean of around 1785 and Joseph Marie Bouton of c. 1800." I put these as additional references in the footnote. Therefore, in the main text I combined the information that there are "einige Miniaturen dieser Art" (some miniatures of this kind) with your remark that the pictures offer "less a voyeuristic peep into this girl's bosom than a promise of touch, over a distance". This was a decision with regard to the flow of the text: is this ok?

Commented [MV16]: See below, my comment in footnote 32 
Wir können die Natur dieses Austausches noch besser verstehen, wenn wir ein noch extremeres Beispiel betrachten, das 1828 von der nordamerikanischen Miniaturistin Sarah Goodridge geschaffen wurde (Abb. 16). Die Darstellung zeigt, von kreppartigem Gewebe umrahmt, eine schön geformte, samtige weibliche Brust, die in leuchtenden Hautfarben auf fast transparentem Elfenbein gemalt ist. Trotz der schmalen Bildmaße hat sie eine gewisse Präsenz, ja es äußert sich geradezu ein selbstreflexives Moment. Ein kleines Muttermal in der Nähe des Brustbeins trägt umso mehr dazu bei, diese Ansicht als Brustporträt zu individualisieren. Was wir hier sehen, ist ein beispielloses Selbstporträt der Künstlerin. ${ }^{33}$

Obwohl wir, als Betrachtende, es jetzt erblicken, sollte uns klar sein, dass dieses Bild ursprünglich von niemandem gesehen werden sollte mit Ausnahme des einzigen Betrachters, für den es gemacht wurde. Eingesetzt in ein rotes Lederetui, dessen Satinfutter denselben Farbton wie die milchige Haut der dargestellten Brüste aufweist, wurde dieses Bild von der Künstlerin ihrem Freund Daniel Webster geschenkt, etwa ein Jahr nach ihrer ersten Begegnung im Jahr 1828. Webster, ein verheirateter Senator aus Massachusetts, stand Goodridge in den darauffolgenden zwei Jahrzehnten mindestens zwölfmal Modell, und in dieser Zeit bestand zwischen ihnen ein reger Briefwechsel. Unlängst hat ein Katalog mit amerikanischen Miniaturen dieses Bild als „Freundschaftsgeschenk“ bezeichnet; was dieses immens private Artefakt offenlegt, ist indes gerade die intime Dimension, die ihre Beziehung gehabt haben muss. ${ }^{34}$ Stellen wir uns vor, was dieses Schmuckstück für Webster bedeutet haben muss, wenn er das Lederetui unterwegs in seiner Tasche trug und es nur in Momenten absoluter Einsamkeit öffnete, um seinen Blick auf eine Kostbarkeit zu richten, die nur ihm gehörte. Die Brüste sind ohne Zweifel ihm angeboten, sie scheinen förmlich nur seiner zu harren. Statt an einen Moment der Intimität zu erinnern, scheinen die Brüste diesen eher zu erwarten: Dieses Geschenk ist eine Einladung zu einer Begegnung, die durchaus nie stattgefunden haben mag - dann wäre diese Miniatur ein Zeugnis unerfüllten Begehrens. Goodridge entschied sich bewusst dafür, Webster ein Bild ihres Körpers statt ihres Gesichts zu schenken. Sie wollte ihm ein Bild eines Teils von

\footnotetext{
${ }^{33}$ Zu der Bildanalyse vgl. hier und im Folgenden auch Grootenboer 2013 (wie Anm. 1), S. 107-108. Zu der Miniatur vgl. auch u.a. Robin Jaffee Frank (Hg.), Love and Loss: American Portrait and Mourning Miniatures (Ausst.-Kat. New Haven, CT, Yale University Art Gallery, Charleston, SC, Gibbes Museum of Art, Andover, MA, Addison Gallery of American Art), New Haven/London 2000, S. 259-264.

Commented [MV17]: Could you add a bibliographical reference? Is it the catalogue "Love and Loss", which I added in footnote 33 , or is it another one? 
ihr geben, den niemand sehen konnte - und da sie nie heiratete, ist es wahrscheinlich, dass niemand außer ihr selbst sie je so gesehen hat. Durch das Öffnen der kleinen roten Schachtel konnte Websters Blick sich sozusagen auf eine Reise in ein unberührtes Territorium begeben und etwas sehen, zu dem niemand außer Goodridge selbst Zugang hatte. Das Geschenk dieses Bildes kann als Triebfeder des Begehrens betrachtet werden, wenn eine tatsächliche körperliche Begegnung unerfüllt bleiben musste. ${ }^{35}$

Goodridge schuf dieses Selbstporträt in dem Jahr, in dem sie 40 Jahre alt wurde. Als ein Geschenk an Webster ist das Bild zugleich auch eine Ode an ihren alternden Körper und als solches eine Liebeserklärung nicht so sehr an ihren Freund wie an die Schönheit ihrer eigenen, noch immer jugendlich aussehenden Brüste. Wenn wir die Sorgfalt studieren, mit der dieses Bild gemalt wurde, die feinen Übergänge von Schneeweiß zu schattigem Grau, die subtilen Schattierungen, die die ganze Fülle ihrer Brüste zum Ausdruck bringen und sie fühlbar werden lassen, können wir nahezu ihrem eigenen zärtlichen Blick folgen, mit dem sie das Spiegelbild ihres Körpers zu streicheln scheint, während sie es malt. Vielleicht ist dies der Grund, warum die Betrachtung dieses Bildes den Eindruck erweckt, dass wir in einen privaten Raum eindringen, und wir das unbehagliche Gefühl bekommen, wir hätten das Etui nicht öffnen sollen. Auf eine bestimmte Art und Weise vermittelt dieses Bild, das heute paradoxerweise mit „Beauty Revealed“ betitelt wird, das Gefühl, dass wir zu viel sehen, dass unser Blick eine Intimität verletzt, die nicht geteilt werden will, sondern nur geraubt werden kann. ${ }^{36}$

Tatsächlich ist die Vorstellung des Intimen im Bezug auf den Blick als ein Raum definiert, in dem man vor öffentlicher Exponierung geschützt ist, in den kein voyeuristisches Auge eindringen kann. ${ }^{37}$ Websters exklusiver Zugang zur intimen Sphäre des Etuis, ebenso wie der flüchtige Blick auf ein Augen- oder Mundporträt, soll nicht das Sehen von Geheimnissen gestatten, sondern das Sehen im Geheimen. Indem sie eine Zone des Intimen markieren, in der das Öffentliche und das Private sich in konstanter Fluktuation befinden, schaffen Briefe, Porträtminiaturen, Augen-

\footnotetext{
${ }^{35}$ Grootenboer 2013 (wie Anm. 1), S. 108.

${ }^{36}$ Ebd.

${ }^{37}$ Vgl. auch Gérard Wajcman, The Birth of the Intimate I, in: Lacanian Ink 23 (Sommer/Herbst 2004), S. 57-81, hier 75 .
} 
und Mundporträts ebenso wie die Orte, an denen sie aufbewahrt und von ihren Besitzern betrachtet wurden, einen intimen, abgeschiedenen Raum: Hier kann das Bild eines Mundes wie ein Kuss gefühlt werden, kann ein stummes Bildnis sprechen, die Figur im Bild nur für den Empfänger posieren und das Geschenk eines solchen ,Porträts‘ tatsächlich eine Berührung versprechen. ${ }^{38}$

${ }^{38}$ Grootenboer 2013 (wie Anm. 1), S. 108. 